\title{
Partial reinforcement effects in a runway: Between- and within-Ss'
}

WILLIAM B. PAVLIK, PETER L. CARLTON AND RICHARD A. HUGHES

\begin{abstract}
Sixteen rats were given 96 continuous reinforcement (CRF) training trials to one stimulus and 96 partial reinforcement (PR) training trials to a second stimulus. These were followed by 40 extinction trials to each of the stimuli. Control groups $(\mathrm{N}=6)$ were given either 96 CRF or 96 PR training trials, followed by 40 extinction trials. During extinction, a conventional partial reinforcement effect (PRE) was found for both start and goal speed measures for the control groups. The within-Ss comparisons yielded no effect of schedules for start speed, but a significant reversed PRE for goal speed.

\section{Introduetion}

It has recently been reported (Pavlik \& Carlton, in press) that rats trained in a free operant situation on continuous reinforcement (CRF) in the presence of one stimulus and on partial reinforcement $(P R)$ in the presence of a second stimulus, when extinguished in the presence of both stimuli (alternately), gave more responses during the CRF stimulus than the PR stimulus. This constitutes a reversal of the conventional partial reinforcement effect (PRE). Control groups trained with only a single schedule yielded a conventional PRE.

Amsel et al (1964; Amsel, 1964) also have used the within-Ss design in studying the PRE, but in a runway situation. During acquisition, the same pattern of results have been obtained from both within- and between-Ss designs; PR eventually produced faster running than did CRF for starting speed measures, but not for goal speed measures. The extinction data reported by Amsel yielded a conventional PRE between-Ss, but no effect of schedules within-Ss; the within-Ss group ran as fast during extinction in both schedules as did a PR control group.

In view of the different extinction results of the studies mentioned above, the present experiment was undertaken to attempt duplicating our free operant situation results in a runway situation.

\section{Method}

Subjects. The Ss were 28 naive male Sprague-Dawley rats which weighed between 180 and 200 gmat the start of the experiment.

Apparatus. The apparatus was a 6-ft runway connecting a 12-in start with a 10-in goal box. All parts were 3 in wide and 5 in high and were painted flat black. The tops of the start box and runway were opaque, that of the goal box transparent plexiglas. Plexiglas guillotine doors separated the runway from the start and goal boxes. The rear wall of the goal box was formed of translucent plexiglas, behind which was mounted a $7-1 / 2$ w bulb. Illumination of this bulb provided bright, diffuse illumination of the goal box ("Bright" goal box). When this light was not lit, the goal box was dimly illuminated from a $15 \mathrm{w}$ bulb suspended $5 \mathrm{ft}$ above the goal box ("Dark" goal box)。

Photoelectric circuitry provided two measures of running speed. The first, Start Speed, was measured from the opening of the startbox door to the intersection of a beam 30 in into the runway. The second, Goal Speed, covered a distance of $4 \mathrm{ft}$, from a beam 30 in from the start box to a beam in the goal box, located 4 in in front of the food cup.

Procedure. All Ss were maintained on a deprivation regimen of $10 \mathrm{gm}$ of Purina Chow per day, which started 10 days prior to training and continued throughout the experiment. Water was always available in the home cage.

Groups CRF and PR $(\mathrm{N}=6)$ each received 96 acquisition trials, at four/day. Group CRF was reinforced on each trial, Group PR twice in each day's block of four trials. Half the Ss in each group were run to the Bright and half to the Dark goal box.

Group Mult $(\mathrm{N}=16)$ received 192 acquisition trials (96 with CRF and 96 with PR) at eight/day, four/day with CRF and four/day with PR. Half the Ss in Group Mult had CRF associated with the Bright goal box and PR with the Dark goal box; the reverse correlation of schedule with goal box obtained for the other half of the Ss. Schedules and goal box stimuli were counterbalanced across Ss and trials such that, for any given ordinal trial, half the Ss were running to each goal box, half running to a given box were on CRF, the other half on PR, and half of those on PR were reinforced and half not. Each reinforcement consisted of five 45-mg Noyes pellets. The intertrial interval was approximately $2 \mathrm{~min}$.

During extinction, each $\mathrm{S}$ was treated exactly as during acquisition except that no reinforcements were given. Groups CRF and PR received 40 extinction trials, at five/day. Group Mult received 80 extinction trials (40 to each goal box) at eight/day, four/day to each goal box.

\section{Results}

All time measures were converted to speed scores (1/1at.), and a mean speed score for each measure was computed for each $\mathrm{S}$ for two day blocks. For Ss in Group Mult, two such means were computed, one for each schedule.

The principal data are presented in Fig. 1 and 2 , which present group means for Start and Goal Speeds, 


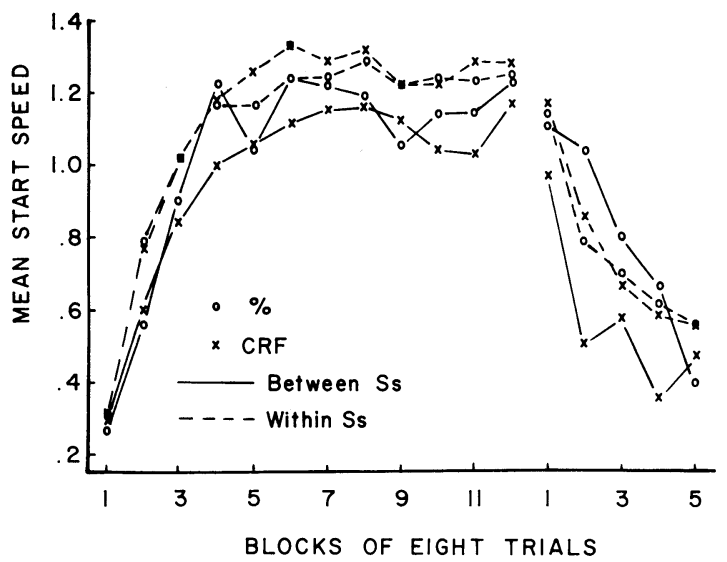

Fig. 1. Mean Start Speed during acquisition and extinction.

respectively, over blocks of two days. During acquisition, the Start Speed measure yielded a between-Ss "crossover effect," in which Group PR initially ran more slowly than Group CRF, but eventually surpassed Group CRF. With only six Ss per group, however, the groups did not differ significantly on the last block of acquisition trials $(p=.12$ by the Mann-Whitney test). Group Mult, in contrast, typically ran faster to the CRF goal box than to the PR goal box. Although the difference is small, the last block of acquisition trials yielded a significant effect of schedules, $\mathrm{p}=.05$ by Wilcoxon's test. This finding is just the opposite of that reported by Amsel et al (1964). Goal Speed yielded no significant differences during acquisition.

During extinction, Start Speed yielded a significant conventional PRE between Groups $\mathrm{CRF}$ and PR, $F(1,10)=10.72, p<.01$. No effect of schedules was apparent for Start Speed in Group Mult; as Fig.1 indicates, Group Mult performed as a group trained on $75 \%$ random reinforcement might be expected to perform. Across design comparisons of mean speed during extinction revealed no difference between the performance of Group PR and the PR performance of Group Mult, but significantly slower running by Group CRF than by Group Mult in the CRF condition $(p<.05$ by the Mann-Whitney test).

Goal Speed during extinction (see Fig. 2) yielded a significant conventional PRE between Groups CRF and PR, F $(1,10)=31.05, p<.001$. Group Mult, however, yielded a reversed PRE; a Type VII analysis of variance (Lindquist, 1953, p.297) yielded a significant effect of schedules, $F(1,14)=9.19, p<.01$. In the latter comparison, 13 of the 16 Ss ran faster to the goal box associated with CRF than to the goal box associated with PR ( $p<.05$ by Wilcoxon's test). Across design comparisons, using the Mann-Whitney test, show that Group Mult ran faster in CRF than did Group CRF $(p=.002)$ and

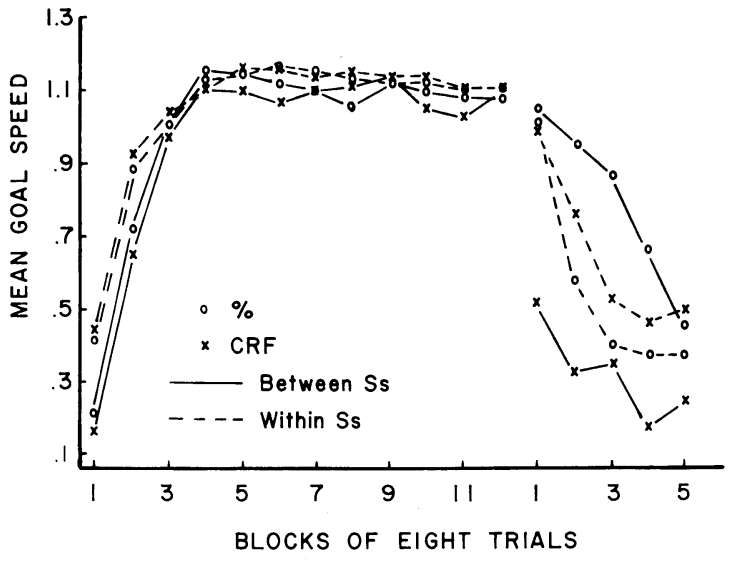

Fig. 2. Mean Goal Speed during acquisition and extinction.

slower in PR than did Group PR $(p=.02)$. The latter difference is consistent with the free operant results of Pavlik \& Carlton (in press).

\section{Diseussion}

Of the various results of the present experiment, only the Start Speed extinction results, showing that Group Mult ran faster in both schedules than a CRF control group, but not significantly slower than a PR control group, are consistent with the results of Amsel et al (1964; Amsel, 1964)。Our acquisition results and extinction Goal Speed results differ from theirs, but are in substantial agreement with our previous findings in the free operant situation.

Just why our results do not confirm those of the Amsel studies is not apparent. The studies do differ in several respects-number and distribution of trials, magnitude of reward, the particular stimuli associated with the schedules-and it may be that at least one of the sets of results may be tied to a narrow range of conditions. Nevertheless, the finding of a reversed PRE during extinction for the Goal Speed measure in the present study, coupled with the reverse PRE in the free operant situation previously reported, do suggest that the conventional PRE may not be as ubiquitous as previously thought, especially in within-Ss designs.

\section{References}

Amsel, A., Mackinnon, J. R., Rashotte, M. E. \& Surridge. C. T. Partial reinforcement (acquisition) effects within subjects. J. exp. Anal. Behar., 1964, 7, 135-138.

Amsel, A. Comparisons of within- and between-subjects partial reinforcement acquisition and extinction effects. Paper read at Psychonomic Society Meetings, Niagra Falls, Oct., 1964.

Lindquist, E, F. Design and analysis of experiments in psychology and education. Boston: Houghton-Mifflin, 1953.

Pavlik, W. B., \& Carlton, P. L. A reversed partial reinforcement effect. J. exp. Psychol., in press.

Note

1. This research was supported by PHS Grant 07844. Portions of this paper were presented at the 1964 meetings of The Psychonomic Society. 